\title{
Dynamic Power Allocation for Multi-hop Linear Non-regenerative Relay Networks
}

\author{
Tingshan Huang, Wen Chen, and Jun Li \\ Department of Electronics Engineering, \\ Shanghai Jiaotong University, \\ Shanghai, China 200240 \\ \{ajelly_172;wenchen;jleesr80\}@sjtu.edu.cn .
}

\begin{abstract}
In this paper, we analyze the optimal power allocation for the multihop linear nonregenerative relay cooperative system. The expression for the end-to-end SNR is deprived in terms of power allocation factors to each node and the channel state information, based on which, we obtain the optimal power allocation factors for each nodes by maximizing the end-to-end SNR subject to the total power constraint. Numerical simulations show that the theoretical prediction matches the simulation, and dynamic power allocation outperforms the fixed power allocation scheme.
\end{abstract}

Index Terms-multihop linear relay networks, amplify-andforward, end-to-end SNR, power allocation.

\section{INTRODUCTION}

By relaying information from the source node to the destination node through a number of intermediate terminals, performance of the communication system is improved significantly in terms of capacity, coverage and reliability. The deployment of relays allows mobility to a further extent by enabling network connectivity where traditional networks may fail to work due to location constraints. Besides, it requires less transmit power to apply multihop relays into the communication system. Therefore, the relaying technique is widely applied, such as in the next generation cellular networks, wireless local area networks, and hybrid networks. Furthermore, the researches on multihop relays attract interest in cooperative communication, where the mobile users cooperate/collaborate with each other in order to exploit the benefits of spatial diversity without the need for using physical antenna arrays [1], [2], [3], [4], [5].

Previous analysis on the end-to-end SNR for relay network can be found in [1], [2], [3], [4]. In [1] Hasna and Alouini have presented end-to-end performance of two-hops wireless communication systems with nonregenerative relays over flat Rayleigh-fading channels, and in [2] they present a general analytical framework for the evaluation of the end-to-end outage probability of multihop wireless communication systems with nonregenerative relays channel sate information assisted relays over Nakagami fading channels. In [3], Karaginannidis has studied the performance bounds for multihop relayed transmissions with blind (fixed-gain) relays over Nakagami$n$ (Rice), Nakagami- $q$ (Hoyt), and Nakagami- $m$ fading channels. Later in [4], Karaginannidis has present a closed-form lower bounds for the performance of multihop transmissions

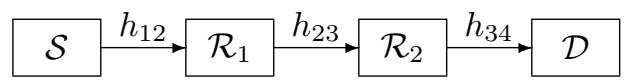

Fig. 1. Illustration of the multihop wireless network when two relays are deployed

with nonregenerative relays over not necessarily identically distributed Nakagami- $m$ fading channels.

Meanwhile, lots of work has been done on power allocation for the different multihop schemes [6], [7], [8]. In [6], Zhao et al present an optimal power allocation scheme to minimize the outage probability for an Amplify-and-Forward (AF) cooperative diversity system. In [7], Bhatia and Kodialam studied the joint routing, scheduling and power control problem for multi-hop wireless parallel relay networks. In [8], Neely et al consider dynamic routing and power allocation for a wireless network with time-varying channels. On the other hand, most of the traditional power allocation schemes [1], [2], [3], [4], assume that power resources are equally distributed over all nodes. The equally power allocation among all transmitters is clearly sub-optimal.

In this paper, we first derive the expression of end-toend SNR for the multihop linear relay network, and then present a power allocation scheme to maximize the end-to-end SNR in an arbitrary-hop cooperative communication system. The proposed power control mechanism varies the transmit power at different transmit nodes based on their corresponding instantaneous channel condition. Different from previous work in [2], which simply normalized the signals received at each transmit node, we amplify those signals at each node according to their upcoming channel states, and make the value of the end-to-end SNR less affected by the noise along the upcoming link but more reliable on the transmit power allocated to each transmit node.

\section{SySTEM MODEL}

In this paper, we consider a wireless linear network with the deployment of $m$ relays. This communication system consists of a source node $\mathcal{S}$ and a destination node $\mathcal{D}$, and the intermediate relay nodes $\mathcal{R}_{1}, \mathcal{R}_{2}, \cdots \mathcal{R}_{m}$ placed on the line between $\mathcal{S}$ and $\mathcal{D}$. An illustration of our model when two relays are deloyed is shown in Fig. 1. 
In our discussion, the system uses the relaying mode of $\mathrm{AF}$, a nonregenerative relaying method that helps to eliminate the effects of delay. In our deployment of AF, the signal received by the $(k+1)$-th relay $\mathcal{R}_{k+1}$ from the previous node, either $\mathcal{R}_{k}$ or the source node $\mathcal{S}$, is first normalized to unity and then amplified along with the noise, and retransmitted to the next first relay along the transmit routine, either $\mathcal{R}_{k+2}$ or the destination node $\mathcal{D}$. The nongenerative method of relaying aims to invert the fading state of the preceding channel, while limiting the instantaneous output power of the relay if the fading amplitude of the preceding hop is low [3]. One complex process is to fix the transmit power at each node, as to be discussed later. We also assume here that neither direct transmission nor other forms of indirect transmission is considered in our discussion.

In this paper, we assume the availability of perfect channel state information at all the nodes in the network. This requirement is necessary, and the reason is indicated in the following part, where the expression for the distribution of the total transmit power among different nodes is given.

In addition, all channels are assumed to be flat Rayleighfading and the channel gains are mutually independent with unit variance. In figure $1, h_{12}, h_{23}$ and $h_{34}$ are the random, complex-valued, unit-power channel gain between source and the first relay, channel gain between the first relay and the second relay, channel gain between the second relay and the destination respectively, and $h_{12}, h_{23}$ and $h_{34}$ are mutuallyindependent and non-identical. $n_{1}, n_{2}$ and $n_{3}$ all are additive Gaussian white noise of the channel $\mathcal{S} \longrightarrow \mathcal{R}_{1}, \mathcal{R}_{1} \longrightarrow$ $\mathcal{R}_{2}, \mathcal{R}_{2} \longrightarrow \mathcal{D}$ respectively. $n_{1}, n_{2}$ and $n_{3}$ all are zeromean, mutually independent, circularly symmetric and white complex Gaussian, obeying the distribution of $\mathcal{C N}\left(0, N_{0}\right)$. Assumptions are the same with the linear network with more than two hops. The total transmit power of the communication system is denoted by $P_{t}$.

\section{END-TO-END SNR ANALYSIS}

In this section, we derive the expressions for the end-toend SNR with power allocation factors in the multihop linear relay system. Since similar work has been done in [2], Our derivation just mention the difference parts.

\section{A. The two-relay systems}

At first we analysis the end-to-end SNR with power allocation factors for the cooperative system where two relays are deployed.

Theorem 1: Suppose that the power allocation factors for nodes $\mathcal{S}, \mathcal{R}_{1}, \mathcal{R}_{2}$ are respectively $E_{1}, E_{2}$ and $E_{3}$. The endto-end SNR of the overall link $\mathcal{S} \longrightarrow \mathcal{R}_{1} \longrightarrow \mathcal{R}_{2} \longrightarrow \mathcal{D}$ is given by

$$
S N R=\frac{\rho_{1} \rho_{2} \rho_{3}}{1+\rho_{1}+\rho_{2}+\rho_{3}+\rho_{1} \rho_{2}+\rho_{1} \rho_{3}+\rho_{2} \rho_{3}},
$$

where $\rho_{1}=\left|h_{12}\right|^{2} \frac{E_{1}}{N_{0}}$ is the instantaneous SNR of the relayed signal from $\mathcal{S}$ to $\mathcal{R}_{1}, \rho_{2}=\left|h_{23}\right|^{2} \frac{E_{2}}{N_{0}}$ is the instantaneous SNR of the relayed signal from $\mathcal{R}_{1}$ to $\mathcal{R}_{2}$, and $\rho_{3}=\left|h_{34}\right|^{2} \frac{E_{3}}{N_{0}}$ is the instantaneous SNR of the further relayed signal from $\mathcal{R}_{2}$ to $\mathcal{D}$.

Proof: At the beginning, the symbol transmitted by the source terminal at its second time slot is denoted as $y_{1}=x$. The data symbols $x$ may be chosen from a complex-valued finite constellation such as quardrature amplitude modulation or from a Gaussian codebook, with the quality of $\mathcal{E}(x)=0$ and $\mathcal{E}\left(|x|^{2}\right)=1$.

At each relay, the received signal is amplified edition of the signal send by the previous node, with the gain denoted by $g_{i}=\sqrt{E_{i}} h_{i, i+1} /\left|y_{i}\right|$ and the received signal is given by

$$
\begin{aligned}
y_{i+1} & =\sqrt{E_{i}} h_{i, i+1} \frac{y_{i}}{\left|y_{i}\right|}+n_{i} \\
& =g_{i} y_{i}+n_{i},
\end{aligned}
$$

where $E_{i}$ is the average transmitted signal energy of the $(i-$ 1)-th relay node over one symbol period. Thus we have the expression for the average energy for the useful signal

$$
E=\frac{\rho_{1} \rho_{2} \rho_{3}}{\rho_{1} \rho_{2}+\rho_{1}+\rho_{2}+1} N_{0},
$$

and the average energy for the noise received at the destination node is

$$
N=\frac{1+\rho_{1}+\rho_{2}+\rho_{3}+\rho_{1} \rho_{2}+\rho_{1} \rho_{3}+\rho_{2} \rho_{3}}{\rho_{1} \rho_{2}+\rho_{1}+\rho_{2}+1} N_{0} .
$$

Thus we have the expression for the end-to-end SNR with power allocation factors as (1).

\section{B. The multihop systems}

For extension, we deduce a more general expression for the end-to-end SNR of the cooperative communication with $m$ relays.

Theorem 2: The end-to-end SNR in the $m$-relayed communication system is given by

$$
S N R^{\{m\}}=\frac{\prod_{i=1}^{m+1} \rho_{i}}{1+\sum_{j=1}^{m} \sum_{i_{p} \neq i_{q}, i_{k} \leq m+1} \prod_{k=1}^{j} \rho_{i_{k}}} .
$$

Proof: We use the mathematical induction to deduce our result. Obviously, the theorem holds for the cooperative systems with two relays by Theorem 1 . Suppose that the theorem is valid in an $n$-relayed communication system. In an $(n+1)$-relayed communication system, the signal transmit by the last relay is denoted as $y_{n+2}=A x+B$, where $A$ represent the useful part in the received signal and $B$ represent the noise. Then we have the average energy for the useful signal at the last relay,

$$
\mathcal{E}\left\{|A|^{2}\right\}=E^{\{n\}}=\frac{\prod_{i=1}^{n+1} \rho_{i}}{1+\sum_{j=1}^{n} \sum_{i_{p} \neq i_{q}, i_{i} \leq n}^{j} \prod_{k=1}^{j} \rho_{i_{k}}} .
$$


The average energy of noise at the destination node is

$$
\mathcal{E}\left\{|B|^{2}\right\}=N^{\{n\}}=\frac{1+\sum_{j=1}^{n} \sum_{i_{p} \neq i_{q}, i_{k} \leq n+1} \prod_{k=1}^{j} \rho_{i_{k}}}{1+\sum_{j=1}^{n} \sum_{i_{p} \neq i_{q}, i_{i} \leq n}^{j} \prod_{k=1}^{j} \rho_{i_{k}}} .
$$

Meanwhile, we also have

$$
\mathcal{E}\left\{\left|y_{n+2}\right|^{2}\right\}=E^{\{n\}}+N^{\{n\}} .
$$

Then the signal received by the destination node is given by

$$
y_{n+3}=\sqrt{E_{n+2}} h_{n+2, n+3} \frac{y_{n+2}}{\left|y_{n+2}\right|}+n_{n+2} \text {. }
$$

Therefore, the average energy for the useful signal at the destination node in the $(n+1)$-relayed communication system is given by

$$
E^{\{n+1\}}=\frac{|A|^{2} E_{n+2}\left|h_{n+2, n+3}\right|^{2}}{\left|y_{n+2}\right|^{2}},
$$

and the average energy for the noise at the destination node in the $(n+1)$-relayed communication system is

$$
N^{\{n+1\}}=\frac{|B|^{2} E_{n+2}\left|h_{n+2, n+3}\right|^{2}}{\left|y_{n+2}\right|^{2}}+N_{0},
$$

where we denote $\rho_{n+2}=\left|h_{n+2, n+3}\right|^{2} \frac{E_{n+2}}{N_{0}}$ as the instantaneous SNR at the last relay node. It implies that the end-to-end SNR for $(n+1)$-relayed cooperative system is

$$
S N R^{\{n+1\}}=\frac{E^{\{n+1\}}}{N^{\{n+1\}}}=\frac{|A|^{2} \rho_{n+2} N_{0}}{|B|^{2} \rho_{n+2} N_{0}+\left|y_{n+2}\right|^{2} N_{0}} .
$$

Inserting (6) and (7) into the above equation, the proof is completed.

The form of equation (5) is similar to equation 2 in [2]. But we have $\rho_{i}=\left|h_{i, i+1}\right|^{2} \frac{E_{i}}{N_{0}}=\gamma_{i} E_{i}$ instead of $\gamma_{i}$ in equation (5), where $\gamma_{i}=\left|h_{i, i+1}\right|^{2} / N_{0}$ denotes the SNR at each node. This difference allows the application of power allocation. In other words, the performance of the cooperative communication in terms of end-to-end SNR can be improved by adjusting the power allocation factors $E_{i}$ in equation (5). Since the addictive noise and channel conditions are fixed in one system model, the optimal power allocation is trying to get the highest endto-end SNR described by equation (5) subject to the power constraint $\sum_{i=1}^{m+1} E_{i}=P_{t}$.

\section{Power Allocation Scheme}

In general, the energies available at the source and the relay nodes in the communication system are constrained by a total energy and a per-node energy constraint. Therefore, our optimization process for power allocation scheme is modeled to achieve the highest end-to-end SNR. Besides, the maximization of the end-to-end SNR can lead to the maximum instantaneous capacity along the link. The optimal power allocation can also lead to a maximum decrease in the outage probability.
The process of optimization with the expression of end-toend SNR is described as follows:

$$
\begin{aligned}
& {\left[\begin{array}{llll}
E_{1} & E_{2} & \cdots & E_{m+1}
\end{array}\right]_{o p t}=\arg \max _{\sum_{i=1}^{m+1} E_{i}=P_{t}}\left\{S N R^{\{m\}}\right\}} \\
& =\arg \max _{\sum_{i=1}^{m+1} E_{i}=P_{t}}\left\{\frac{\prod_{i=1}^{m+1} \rho_{i}}{1+\sum_{j=1}^{m} \sum_{i_{p} \neq i_{q}, i_{k} \leq m+1} \prod_{k=1}^{j} \rho_{i_{k}}}\right\} .
\end{aligned}
$$

Although the expression in (5) enables numerical evaluation of the system performance, it results in intensive computation. Considering the complexity of the expression, we make an approximation and the equation (5) becomes

$$
S N R^{\{m\}}=\frac{\prod_{i=1}^{m+1} \rho_{i}}{\sum_{i_{p} \neq i_{q}, i_{k} \leq m+1} \prod_{k=1}^{j} \rho_{i_{k}}},
$$

which lead to

$$
\frac{1}{S N R\{m\}}=\sum_{i=1}^{m+1} \frac{1}{\rho_{i}}=\sum_{i=1}^{m+1} \frac{1}{A_{i} E_{i}}
$$

where $A_{i}=\left|h_{i, i+1}\right|^{2}$.

The approximation of the end-to-end SNR made above is accurate enough at moderate and high SNR values, and as a result, helps to facilitate the process of optimization. As a matter of fact, it is proved in [?] that the form of the equivalent SNR in (15) can be looked as if it is arising from an ideal/hypothetical relay that is able to invert the channel regardless of its magnitude and with a gain given by $g_{i}=\sqrt{E_{i}} h_{i, i+1}$ instead of $g_{i}=\sqrt{E_{i}} h_{i, i+1} /\left|y_{i}\right|$.

Now, the maximization of the end-to-end SNR turns out to be the process of minimization of $\frac{1}{S N R^{\{m\}}}$, i.e.,

$$
\begin{aligned}
& {\left[\begin{array}{llll}
E_{1} & E_{2} & \cdots & E_{m+1}
\end{array}\right]_{\text {opt }}=\arg \min _{\sum_{i=1}^{m+1} E_{i}=P_{t}}\left\{\frac{1}{S N R^{\{m\}}}\right\}} \\
& =\arg \min _{\sum_{i=1}^{m+1} E_{i}=P_{t}}\left\{\sum_{i=1}^{m+1} \frac{1}{A_{i} E_{i}}\right\},
\end{aligned}
$$

Now, we use the Lagrange multipliers to find the solution, and the target function is given by

$$
\begin{aligned}
f & =\frac{1}{S N R^{\{m\}}}+\lambda\left(\sum_{i=1}^{m+1} E_{i}-P_{t}\right) \\
& =\sum_{i=1}^{m+1} \frac{1}{A_{i} E_{i}}+\lambda\left(\sum_{i=1}^{m+1} E_{i}-P_{t}\right) .
\end{aligned}
$$

And solution for the equations set

$$
f^{\prime}\left(E_{k}\right)=\frac{\partial f}{\partial E_{k}}=0, \quad k=1,2, \cdots, m+1,
$$


is given by

$$
E_{k}=\frac{P_{t}}{\sqrt{A_{k} \lambda}}, \quad k=1,2, \cdots, m+1,
$$

and

$$
\sum_{i=1}^{m+1} E_{i}-P_{t}=0
$$

Then we get the value for $\lambda$ as

$$
\lambda=\left(\sum_{i=1}^{m+1} \frac{1}{\sqrt{A_{i}}}\right)^{2} .
$$

Thus the power allocation is expressed by

$$
E_{k}=\frac{P_{t}}{\sqrt{A_{k} \sum_{i=1}^{m+1} \frac{1}{\sqrt{A_{i}}}}}, \quad k=1,2, \cdots, m+1 .
$$

Therefore, we obtain the power allocation scheme as summarized in the following theorem.

Theorem 3: For a multihop linear nonregenerative relay network, the power allocation factors are

$$
E_{k}=\frac{P_{t}}{\sqrt{A_{k} \sum_{i=1}^{m+1} \frac{1}{\sqrt{A_{i}}}}}, \quad k=1,2, \cdots, m+1 .
$$

This solution implies that the optimal amount of power allocated to each node is a function of the total transmit power $P_{t}$ and all the channel state information along the transmitting link. Therefore, to achieve the highest end-to-end SNR in this cooperative communication system, it is necessary for all nodes to require a global channel information. The global channel state information at all the nodes can be accomplished by ways of information exchange, estimate or feedback, which will be discussed in our future research.

In particular, in a multihop system where two relays are deployed, the power allocation that can achieve high end-toend SNR is given by

$$
\begin{aligned}
& E_{1}=\frac{P_{t}}{1+\sqrt{\frac{A_{1}}{A_{2}}}+\sqrt{\frac{A_{1}}{A_{3}}}}, \\
& E_{2}=\frac{P_{t}}{1+\sqrt{\frac{A_{2}}{A_{1}}}+\sqrt{\frac{A_{2}}{A_{3}}}}, \\
& E_{3}=\frac{P_{t}}{1+\sqrt{\frac{A_{3}}{A_{1}}}+\sqrt{\frac{A_{3}}{A_{2}}}} .
\end{aligned}
$$

\section{NUMERICAL RESULTS}

In this section, we numerically demonstrate the optimal power allocation factors for the linear relay system. In our simunation, we assume that information is transmitted over the linear nonregenerative cooperative network with two relays. We also assume an additive unity noise with variance of $N_{0}=1$, the variances for channel gains are $\mathcal{E}\left\{\left|h_{12}\right|^{2}\right\}=$ $1, \mathcal{E}\left\{\left|h_{23}\right|^{2}\right\}=3$ and $\mathcal{E}\left\{\left|h_{34}\right|^{2}\right\}=1$. We choose the different numbers for the channels to show effectiveness of the proposed power allocation scheme.

First we build Mont-Carlo link-level simulation on our model under instantaneous channel state, and the result is shown in Fig 2 and Fig. 3, where the curve represent the relationship of the average end-to-end SNR and different proportions of power at the three transmit nodes. In Fig 2 and Fig. 3, the $z$-axis represents the average value of the endto-end SNR, the $x$-axis is the proportion of the total transmit power allocated to the source node, the $y$-axis is the proportion of the total transmit power allocated to the first relay, and the rest power is allocated to the second relay along the transmit link.

Fig 2 and Fig. 3 demonstrate that when the state of the transmit channel is as assumed, the highest end-to-end SNR can be achieved. The transmit power allocated to the three transmit nodes are as follows:

$$
\left(\frac{E_{1}}{P_{t}}, \frac{E_{2}}{P_{t}}, \frac{E_{3}}{P_{t}}\right)=(0.3880,0.2240,0.3380) .
$$

When our power allocation scheme is applied, the average amount of power allocated to each node along the transmit link should be

$$
\begin{aligned}
& E_{1}=\frac{P_{t}}{1+\sqrt{\frac{A}{B}}+\sqrt{\frac{A}{C}}}=0.3880, \\
& E_{2}=\frac{P_{t}}{1+\sqrt{\frac{B}{A}}+\sqrt{\frac{B}{C}}}=0.2240, \\
& E_{3}=\frac{P_{t}}{1+\sqrt{\frac{C}{A}}+\sqrt{\frac{C}{B}}}=0.3380 .
\end{aligned}
$$

This numerical result shows that our power control mechanism fits the result of the Mont-Carlo simulation well, and thus can achieve high end-to-end SNR.

As a matter of fact, if we vary the power allocation among the transmit nodes according to the instantaneous fading Rayleigh channel state, the performance of the system can be further improved. In the rest part, we will demonstrate the fact by numerical simulation.

In Fig 4, the $x$-axis represents the constraint amount of power consumed over the entire network $P_{t}$, and the $y$-axis is the achieved end-to-end SNR using our power allocation scheme. In Fig. 4, we also plot the curve of the end-toend SNR versus $P_{t}$ when a fixed power allocation is applied regardless of the changes in the channel state. By comparing the two curves, it is clear to see that the dynamic power allocation outperforms the fixed power allocation.

\section{CONCLUSION}

In this paper, we have analyzed the optimal power allocation for the multihop linear nonregenerative relay cooperative system. The expression for the end-to-end SNR is deprived in terms of power allocation factors to each node and the channel state information, based on which, we obtain the optimal power allocation factors for each nodes by maximizing the 


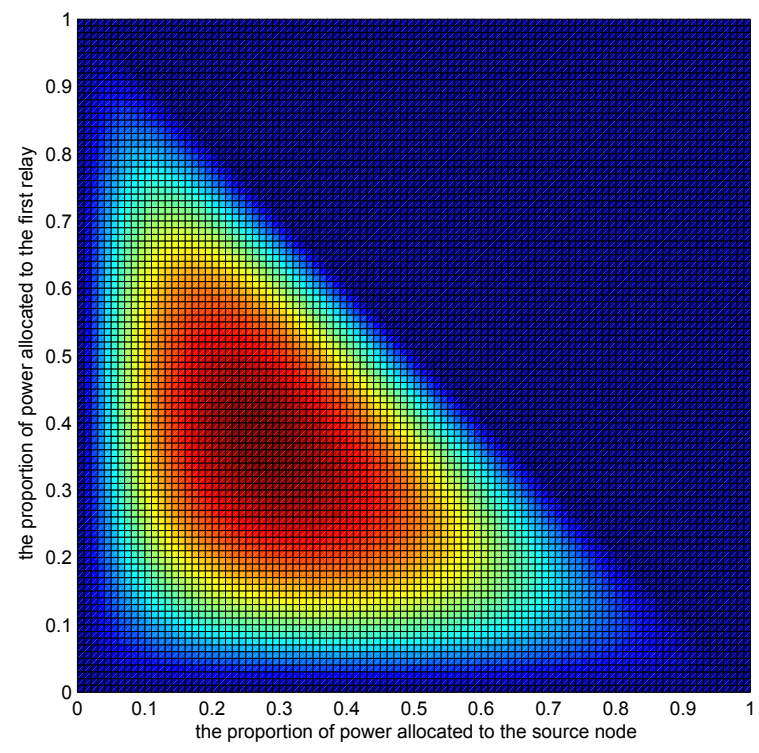

Fig. 2. The average end-to-end SNR under different proportion of power at three transmit nodes, where the color represents the numerical value for the end-to-end SNR, the $x$-axis is the proportion of the power allocated to the source node $\mathcal{S}$ and the $y$-axis is the proportion of power allocated to the first relay $\mathcal{R}_{1}$, while the value of $(1-x-y)$ is the proportion of power allocated to the second relay $\mathcal{R}_{2}$

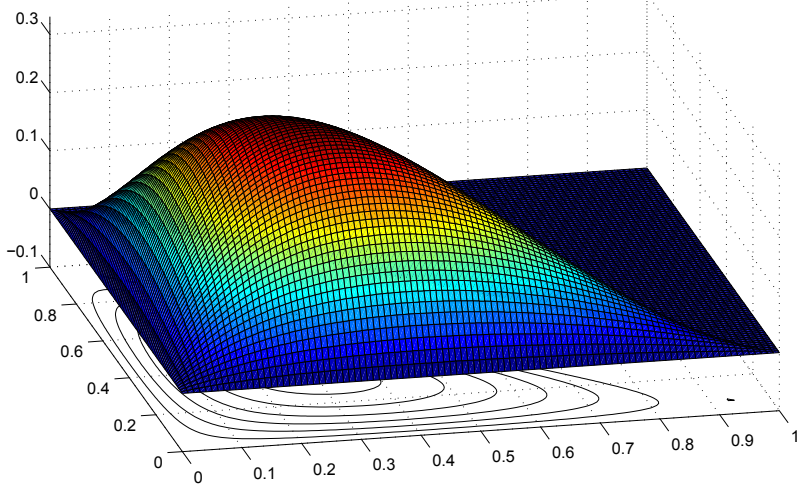

Fig. 3. The average end-to-end SNR under different proportion of power at three transmit nodes, where the $z$-axis represent the numerical value for the end-to-end SNR, the $x$-axis is the proportion of the power allocated to the source node $\mathcal{S}$ and the $y$-axis is the proportion of power allocated to the first relay $\mathcal{R}_{1}$, while the value of $(1-x-y)$ is the proportion of power allocated to the second relay $\mathcal{R}_{2}$

end-to-end SNR subject to the total power constraint. Numerical simulations show that the theoretical prediction matches the simulation, and dynamic power allocation outperforms the fixed power allocation scheme.

\section{ACKNOWLEDGEMENT}

This work is supported by NSF China \#60672067, by NSF Shanghai \#06ZR14041, by Shanghai-Canada NRC

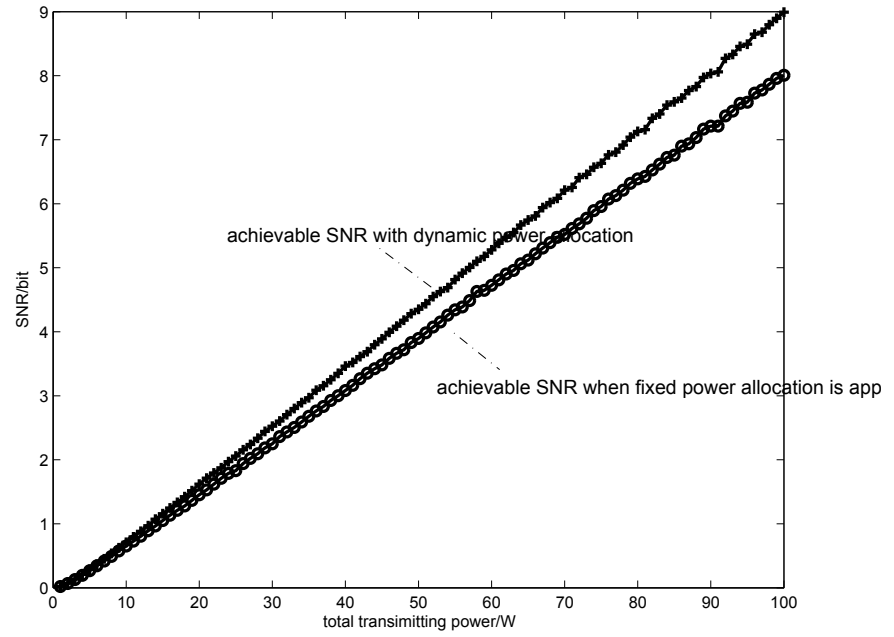

Fig. 4. The end-to-end SNR with the total transmit power in the communication system. The upper curve represents the end-to-end SNR using the dynamic power allocation scheme under instantaneous fading channel, while the lower curve represents the end-to-end SNR using a fixed power allocation of $(0.3880,0.2240,0.3380)$

\#06SN07112, by Cultivation Fund of the Key Scientific and Technical Innovation Project, Ministry of Education of China \#706022, by Program for New Century Excellent Talents in University \#NCET-06-0386, by PUJIANG Talents \#07PJ4046, and by Huawei Fund for Sciences and Technologies in Universities \#YJCB2008048WL

\section{REFERENCES}

[1] M. O. Hasna, and M.-S. Alouini,"End-to-end performance of transmission systems with relays over Rayleigh fading channels," IEEE Trans. Wireless Commun., vol. 2, no. 6, pp. 1126-1131, Nov.2003.

[2] M. O. Hasna, and M.-S. Alouini,"Outage probability of multihop transmission over Nakagami fading channels," IEEE Commun Letters, vol. 7, no. 5, pp. 216-218, May.2003.

[3] G. K. Karagiannidis, "Performance bounds of multihop wireless communications with blind relays over generalized fading channels," IEEE Trans. Wireless Commun., vol. 5, no. 3, pp. 498-503, March 2006.

[4] G. K. Karagiannidis, T. A. Tsiftsis,and R. K. Mallik, "Bounds for Multihop Relayed Communications in Nakagami- $m$ Fading," IEEE Trans. Commun, vol. 54, no. 1, pp. 18-22, Jan. 2006.

[5] J. N. Laneman, D. N. C. Tse, and G. W. Wornell, "Cooperative diversity in wireless networks efficient protocols and outage behavior," IEE Trans. Wireless Commun., vol. 50, no. 12, pp. 3061-3080, Dec. 2004.

[6] Y. Zhao, R. Adve and T. J. Lim, “Improving Amplify-and-Forward Relay Networks: Optimal Power Allocation versus Selection,"ISIT 2006,Seattle, USA, pp. 1234-1238,July 9-14,2006.

[7] R. Bhatia, M. Kodialam, "On power efficient communication over multihop wireless networks: joint routing, scheduling and power control", IEEE INFOCOM 2004.

[8] M. J. Neely, E. Modiano, and C. E. Rohrs, "Dynamic power allocation and routing for time-varying wireless networks",IEEE J. Sel. Areas Commun, vol. 23, no. 1, pp. 89-103, Jan 2005. 